\title{
New ways of working: does flexibility in time and location of work change work behavior and affect business outcomes?
}

\author{
Merle M. Blok ${ }^{\mathrm{a}, *}$, Liesbeth Groenesteijn ${ }^{\mathrm{a}, \mathrm{b}}$, Roos Schelvis ${ }^{\mathrm{a}}$ and Peter Vink ${ }^{\mathrm{a}, \mathrm{b}}$ \\ a, TNO, P.O. Box 718, 2130 AS Hoofddorp, The Netherlands. \\ ${ }^{\mathrm{b}}$ Faculty of Industrial Design Engineering, Delft University of Technology, Landbergstraat 15, 2628 CE Delft, \\ The Netherlands.
}

\begin{abstract}
In the changing modern economy some new factors have been addressed that are of importance for productivity and economic growth, such as human skills, workplace organization, information and communication technologies (ICT) and knowledge sharing. An increasing number of companies and organizations are implementing measures to better address these factors, often referred to as 'the New Ways of Working (NWW)'. This consists of a large variety of measures that enable flexibility in the time and location of work. Expectations of these measures are often high, such as a reduction in operating costs and an increase of productivity. However, scientific proof is still lacking, and it is worth asking whether al these implementations actually cause a change in work behavior and effect business outcomes positively. This article describes a case study of three departments (total of 73 employees) that changed from a traditional way of working towards a new way of working. Questionnaires and a new developed objective measurement system called 'work@task' were used to measure changes in work behavior (i.e. increased variation in work location, work times and a change towards NWW management style) and the effect on business objectives such as knowledge sharing, employees satisfaction, and collaboration.
\end{abstract}

Keywords: new ways of working, task facilitating office, knowledge worker, work behavior, business objectives

\section{Introduction}

The modern economy is changing from agriculture and industrial manufacturing to a service and knowledge driven economy. Knowledge is recognized as the driver of productivity and economic growth, and statistics form the OECD studies show that the number of employees working for knowledge- intensive service sector is increasing [6]. Knowledge work is supported by a revolution in new ICT applications and communication networks. These innovations has changed our perceptions on work and made it possible to work at any location at any time [5]. The proliferating use of information has long been seen as 'the' aspect that would bring us higher productivity and better business outcomes. However aspects such as human talent can be seen of even greater importance, since that makes it possible to share knowledge, adapt and innovate [1]. It is therefore argued that employees, especially knowledge workers, should be more empowered to work more efficiently and effectively [4]. This empowerment implies offering the employees more self control and freedom by introducing flexible work arrangements. This transformation is often referred to as 'the New Ways of Working' (NWW) and consist of changes that take place at four aspects:1) the physical workspace, 2) (ICT) technology, 3) organization \& management and 4) work culture. The physical workspaces refers to NWW measures that increasing the flexibility where and when to work by introducing flexible work hours, telework and creating flexible workplaces at the office that better suits the work task. Introducing ICT technologies implies that employees are supported with technologies that allows them to be connected and able to collaboration always and everywhere. The

*Corresponding author: Merle Blok. E-mail: merle.blok@tno.nl. 
third NWW aspect: 'organization and management' is important since managing employees might become a big challenge when it is not longer visible were, when and what employees are working on. It is therefor important that managers have trust in there employees, focus more on output instead of presence at the office, and provide them with more autonomy by stimulating own initiative. Changes in work culture implies that an open culture, with focus on information sharing and collaboration in networks is created.

Many organizations see potential opportunities in the transition to the NWW and the number of organizations that have implemented a form of NWW is rapidly increasing. This is not only in order to enhance productivity growth, but is also seen as a necessary preparation for the upcoming societal issues. Attracting skilled professionals will get more difficult, since we are facing a demographic shift in aging populations. And there is an increase in road traffic, causing serious traffic infarcts and a loss in productive work time. The NWW measures not only offer differentiation in starting and ending time of work, it also offers the possibility to work from any other remote location. The Telework Trendlines 2009 [7] reported that the number of U.S. employees who worked remotely at least one day per month increased $39 \%$ in two years from approximately 12.4 million in 2006 to 17.2 million in 2008 .

Working from remote locations affect the purpose of the office building, making it less important for the performance of individual work tasks, and more important for work activities such as collaboration, faceto-face meetings and knowledge sharing [2]. To better suit these work activities, a growing number of organization lower the total amount of office building space, and task facilitating offices. This often consists of transparent offices including a large variety of shared workplaces, such as meeting rooms, project places, lounge corners and concentration arias [3].

Although the expectations of the NWW measures are often high, scientific proof is still lacking. It is important to know more about the effects to provide organizations with a better understanding and (at forehand) insight in the effects of their NWW investment or policy decisions regarding the implementation. It is still unknown how implementations of NWW measures affect work behavior, in means of where and when the employees work, and how this relates to business objectives such as increased productivity by improvements in collaboration, knowledge sharing and employee satisfaction.
In this paper a case study is presented of a Dutch organization with a pilot group consisting of three departments that changed from a traditional way of working towards a new way of working. The changes includes a new flexible office layout were workplaces are shared, introduction of social ICT and the ability to work from home or any other remote location at flexible work hours. Their objective was to increase collaboration, knowledge sharing and employees satisfaction, and thereby enhance the productivity of the employees, while at the same time reducing cost by decreasing the amount of total office space used. The effects on work behavior and on the aimed business objectives are monitored every half year for four times in total. A questionnaire and a new developed objective measuring method called 'work@task' to monitor changes in work location are used. The results from the first two measures will be presented in this paper. This article is aimed to provide an answer to the research question: "What are the effects of new ways of working in a task facilitating office on work behavior, and does this positively effect collaboration, employee satisfaction and knowledge sharing?

\section{Method}

A group of 73 employees from three different departments participated in this study. All participants moved from a traditional work environment where each department had his own work space, to one shared work area consisting of a large variety of different shared workspaces such as brainstorm area's, meeting rooms, silent open workspaces and project places. Digital smart boards were introduced to support project work, as well as laptops, cellphones, and access to the business network in order to enable employees to work everywhere throughout the department.

\subsection{Questionnaire}

A web based internet questionnaire was developed and carried out twice, once while implementing the new ways of working (M1), and one six months later in the new office environment (M2). All employees of the three different departments participated in the study. The questionnaire was conducted in order to measure NWW awareness, change in work behavior and the effects on business outcomes. Questions on change in behavior consisted of questions on flexibility in work location and workplace, and if a NWW 
management style was created in the new work environment. Since the first measure (M1) was conducted while at the same time the implementation of the new way of work was implemented, the questionnaire consisted of some questions to retrieved information of the actual stage of the three differed departments, such as habitation to the new flexible work environment.

Questions on NWW management style consisted of items measuring the degree to which managers behaved as a NWW role model, if they listened and showing interest in the work of the employees, and questions on the focus and agreements on results, the feasibility of the results and whether the employees perceived enough autonomy

\subsection{Work@task}

In the new work environment the participant had greater flexibility in the timing and location of work. It was therefore assumed that employees would more frequently change workplaces and work location (at the office, at home, while traveling or at the client office). In order to measure actual behavioral changes in work place and location a 'work@task' system was developed and tested. The method consists of an automatic short message services, were texts massages were send to the business cellphones of sixty employees five times a day at standardized moments in time for a period of two weeks. The employees were asked to respond immediately to each text message with a message code that described their workplace, work location and the task they were performing. In order to make the response as less time consuming as possible, response codes were formulated and profited to the employees at small pocketsize plastic cards (see figure 1) and the workplaces at the office were labeled with code numbers. The work@task measurement was conducted in the new office situation only and corresponded in time with the second questionnaire measure (M2).

\subsection{Statistics}

Descriptive statistics were used to describe the results from the questionnaire and work@task. Withinsubject t-test analysis $(\mathrm{p}<0.05)$ was used on the questionnaire data of participants that participated in both the M1 and M2 questionnaire only, in order to detect significant effects of NWW on collaboration, employees satisfaction and knowledge sharing.

Work@task Codes for short message service
For example O1IC
\begin{tabular}{|l|}
\hline Location \\
\hline O\# = Office + workplace number \\
\hline $\begin{array}{l}\text { OD }=\text { Office, working at a different de- } \\
\text { partment }\end{array}$ \\
\hline $\begin{array}{l}\text { OL }=\text { Working at a different office loca- } \\
\text { tion }\end{array}$ \\
\hline $\mathrm{H}=$ Home \\
\hline $\mathrm{T}=$ Traveling \\
\hline WE = Working extern (at client office) \\
\hline How? \\
\hline $\mathrm{I}=$ individual \\
\hline $\mathrm{T} 1=$ working together at one location \\
\hline T2 = working together at two locations \\
\hline $\mathrm{G} 1=$ group work at one location \\
\hline $\begin{array}{l}\mathrm{G} 2=\text { group work at two or more loca- } \\
\text { tions }\end{array}$ \\
\hline \\
\hline What? \\
\hline $\mathrm{C}=$ concentration task \\
\hline $\mathrm{R}=$ routine task \\
\hline $\mathrm{F}=$ formal meeting \\
\hline $\mathrm{IF}=$ informal meeting \\
\hline $\mathrm{P}=$ Phone call \\
\hline $\mathrm{B}=$ Break \\
\hline $\mathrm{N}=$ Not working \\
\hline
\end{tabular}

Figure1.Work@task codes that were used in the short massage service.

\section{Results}

All 73 employees of the three departments received the first online questionnaire (M1) and half a year later 60 of them received the second questionnaire (M2). In total 58 participants (average age 45; 59\% male) filled out the first questionnaire, while $52 \mathrm{em}$ ployees (average age $44 ; 53 \%$ male) responded to the second questionnaire. A total of 39 participants filled out both questionnaires. The job functions of the subjects existed of either manager, project manager, project support or advisor.

\subsection{Implementation awareness of $N W W$ measures}

Questionnaire data on the status of implementation of the new ways of working and the habituation to the new flexible work layout showed that none of the participants were fully habituated to the new flexible work layout, and a part of the participants $(28 \%)$ were still working at the traditional office at the time the first questionnaire was filled out (M1). Half year later, at the time the second questionnaire (M2) was 
filled out all participants were working at the flexible work layout. More than half $(54 \%)$ of the participants were entirely habituated and $30 \%$ was habituated somewhat. A total of $16 \%$ stated that they were not yet habituated to the new flexible work layout.

In figure 2 the results are shown for differed statements that were addressed in the questionnaire on the possibility to work flexible. The results show an increase over time between M1 and M2 in the experienced possibility to work at flexible work hours at the office, the availability of sufficient ICT facilities and access to business networks from home or other remote work locations. These results indicate that the participant were aware of the new possibilities that were created by introducing the new way of working.

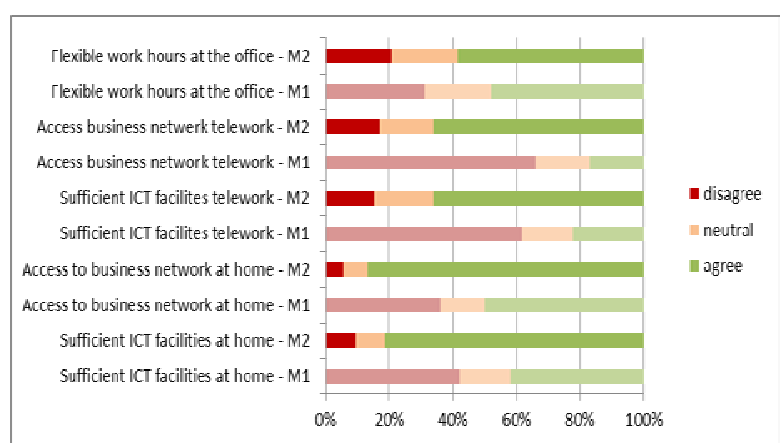

Figure 2. The ability to work flexible in time and the accessibility and sufficient ICT facilities to work from remote locations at measurement M1 $(n=57)$ and M2 $(n=50)$

Besides changes in physical workspace and (ICT) technology, implementations of the NWW also implies changes in organization \& management and a change towards a suitable work culture. The results on NWW management style items of M1 and M2 (see figure 3) show that the overall score on NWW role model and the focus on results improved overtime, although there is still a large percentage of employees that did not experience the manager as a NWW role model (31\%) with forces on results $(15 \%)$. The other aspects of the NWW management style aspect show a decrease over time.

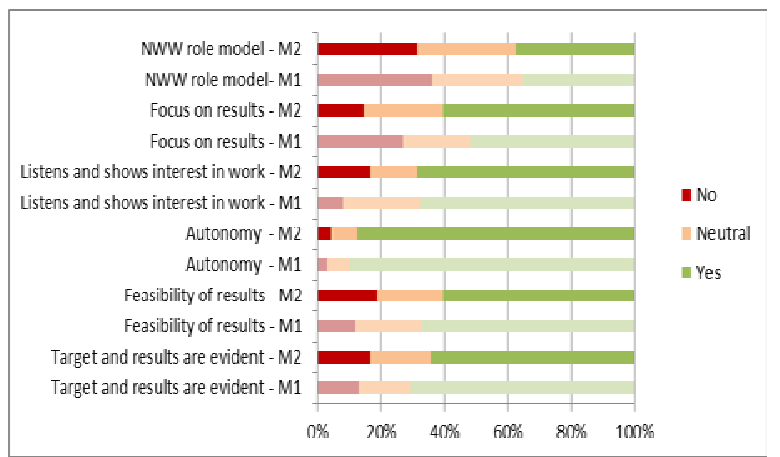

Figure 3. The average score on question items measuring NWW management style $\mathrm{M} 1 \mathrm{n}=48, \mathrm{M} 2 \mathrm{n}=48$.

\subsection{Changes in flexible work behavior}

In order to investigate whether the actual implementation of NWW measures actually caused a change in work behavior the participants were asked where they performed their work tasks. The results in figure 4 show that there were no big changes in amount of working hours spend on different work locations. Working at home increased from 4.5 hours per week at M1 to 5.5 hours at M2, which was not as much as was expected, since at M2 working from home was officially enabled. The biggest increase was seen for working at the client office which increased from 5.8 hours per week to 7.4 hours per week.

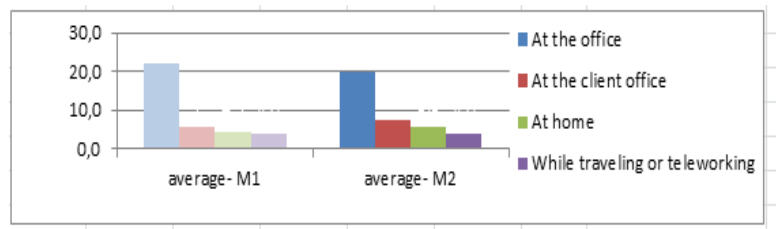

Figure 4. The number of hours per week worked at different locations, at measurement M1 $(n=57)$ and M2 $(n=50)$.

The results from work@task (see figure 5) show that $60 \%$ of the work time was spend at the office building, of which $40 \%$ of the working time was spend at the flexible work layout. A total of $18 \%$ of the working time was spend at home, an another $13 \%$ was spend teleworking extern at the client office. 


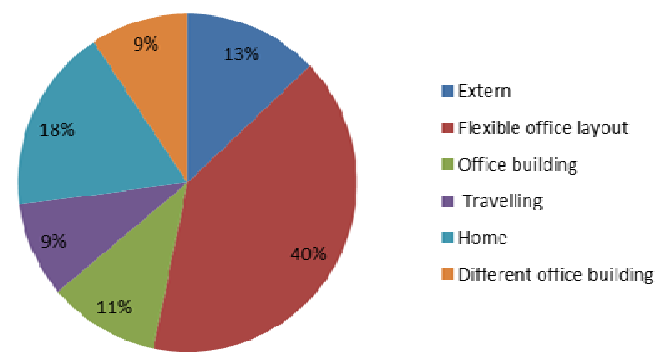

Figure 5. The number of hours per week worked at different locations, at measurement M1 $(n=57)$ and M2 $(n=50)$

At the traditional office the employees had owned workstations, and did not have a variety of different workplaces except for meeting rooms and coffee corners. The new flexible office layout did offer a wide variety of different workspaces (M2). In the work@task measurement the percentage of work time spend at each workplace was measured for M2 (see figure 6). The workplaces at the open area (a total of 31 workplaces), were used for $61 \%$ or the time. The three meeting rooms and team rooms were used $13 \%$ of the time, followed by meeting/lounge rooms. The phone booths were only used $1 \%$ of the time.

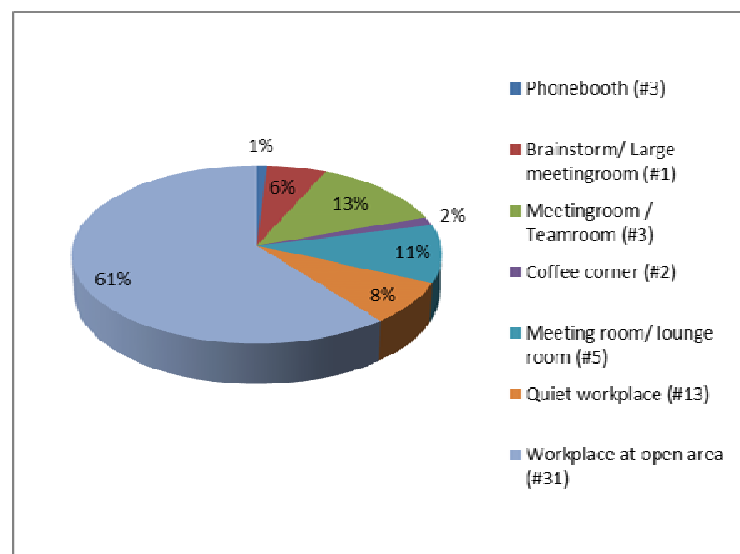

Figure 6. The average number of hours spend at different workplaces at the office for M2 ( $\mathrm{n}=49)$, \# number of workspaces.

\subsection{Effect on business outcomes}

So far, the results have shown that the employees did experience an increase in possibilities to work flexible in time and location and a small change in behavior caused by these increased flexibilities was visible. Results on the business objectives were measured on a scale from 1 'very low' to 7 'very high'. Results did not show any change between M1 and M2 for collaboration and employees' satisfaction and the suitability of the environment to perform the work tasks, while knowledge sharing was decreased significantly (see Fig. 7).

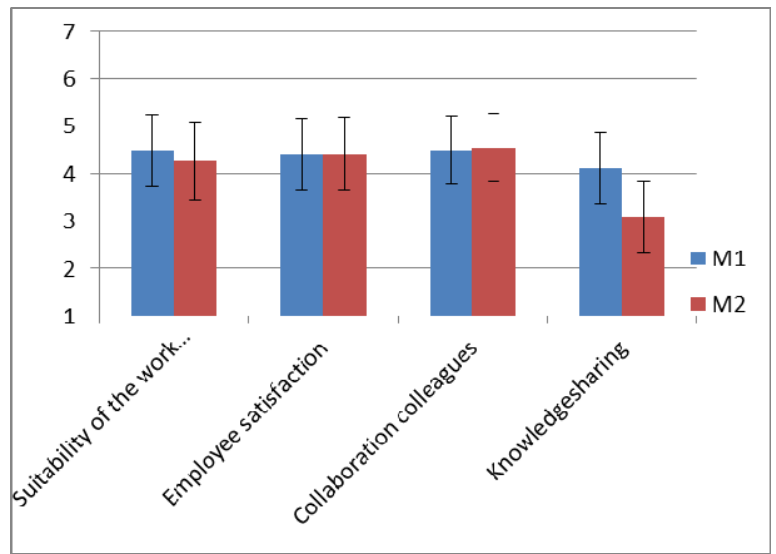

Figure 7. Average scores for M1 and M2 on scale from 1 to 7 ( $1=$ very low, $7=$ very high).

\section{Discussion}

In this research study it was investigated whether the introduction of new way of working measures caused changes in work behavior, leading to positive effects on business objectives. The results of this study showed that the participants were aware of the increased possibility to work at different locations, and they experienced an increase in availability of ICT facilities and better remote access to business networks. It is interesting to see that even after halve a year still not all of the employees were habituated.

Results on the implementation of a NWW management style did not show overall positive results. Four out of six questionnaire items on NWW management style showed a decrease over time. This is a interesting result, since it was expected that the NWW management style was implemented and therefor the experienced NWW management style would improve. It was certainly not expected that it would decrease. This result might indicate that when NWW is introduced the importance of a NWW management style is of greater importance, which might created increased awareness of the absence of NWW management style resulting in lower scores.

As mentioned before, NWW consist of changes that 
take place at four aspects, the physical workspace, (ICT) technology, organization \& management and work culture. From the results we might conclude that at least two out of four NWW aspects (i.e. physical workspace and ICT technologies) were successfully implemented. The implementation of management style was not conducted successfully yet, and should be given more priority. Changing the organizational culture might be of greater effort and take up more time. It will be interesting to see if improvements are seen at a later stage in the third or fourth measure.

Studying the results on change in behavior, some indications are found for the hypothesis that implementing NWW measures changes the work behavior. For instance, more different work locations and workplaces throughout the office were used. It is expected that there will be a greater change in work behavior when all four NWW aspects are implemented successfully.

Not finding any improvements in the business objectives can have at least two important reasons. First of all it can be explained by the fact that not all four aspects of NWW are implemented well enough to cause a significant change in work behavior, and therefore the business objective are not affected. Second of all it is possible that although expected by NWW believers, the NWW measures do not affect of improve the selected business objectives. The NWW might increase ad hoc interaction and communication of colleagues, but this does not imply improvements in knowledge sharing or collaboration Even if knowledge sharing and collaboration at the office itself improves, this might be counteracted by the fact that more time is spend working at home or at other remote locations where less ad hoc interaction and communication has takes place.

This case study provides us with some interesting insights in some of the effects of the NWW measures. It is difficulty to set up a good research study to measure the effects of the NWW since in reality it is difficult to isolate the effects of NWW in organizations, and other changes that might affect the results as well are often taking place as well. In order to gain good inside in the effect of NWW intervention it is important to measure the situation some time before the implementation takes place and a period of time after, when al the short term effects caused by the change toward the NWW measures has disappeared. Unfortunately in this study at the moment of the M1 measure the implementation was already partly started and some of the employees had already moved to the new flexible office layout a few days prior to the measure. Even so, it was not expected that the recent movement did cause an immediate change in business objectives and it is expected that when employees get more habituated to the flexible work environment it will have a positive effect on knowledge sharing, collaboration, satisfaction and experienced suitability of the work environment.

Further research on this topic will be done, since two other measures will be performed. It will be interesting to see whether all four NWW aspects will be further implemented successfully. And if the behavior of the employees will change towards a more flexible work behavior such as a further increase in hours worked at home or remote, changes in work time and more flexibility in the use of different workplaces at the office. It will then be possible to see if a further increase in work behavior will significantly improve the business objectives.

\section{References}

[1] S. Aravaniti and E.N. Loukis, ICT, human capital, workplace organization and labour productivity: A comparative study based on firm-level data for Greece and Switzerland, Information, Economics and Policy 21, 2009, pp. 43-61.

[2] M. Blok, E. de Korte., L. Groenesteijn, M. Formanoy, P. Vink, The effects of a task facilitating working environment on office space use, communication, concentration, collaboration, privacy and distraction. Proceedings of the 17th World Congress on Ergonomics, Bejing, 2009.

[3] E.M. de Croon, J.K. Sluiter, P.P.F.M. Kuijer, and M.H.W. Frings-Dresen, The effect of office concepts on worker health and performance: a systematic review of the literature, Ergonomics, 48: 2, 2005, pp. 119-34.

[4] C. Ichniowski, T.A. Kochan, D.I. Levine, C. Olson, G. Strauss, What works at work: overview and assessment. In: Ichniowski, C., Levine, D.I., Olson, C., Strauss, G. (Eds.), The American Workplace. Skills compensation and Employee Involvement. Cambridge University Press, Cambridge, 2000 pp. 1-37.

[5] S.Y. Lee, J.L. Brand, Effects of control over office workspace on perceptions of the work environment and work outcomes, Journal of Environmental Psychology, 25, 2005, pp.323-333.

[6] OECD, Organization for economic co-operation and development.. The knowledge-based economy. General distribution OCDE//GD(96)102, 1996

[7] WorldatWork Research. Flexible Work Arrangements for Nonexempt Employees, 2009. WorldatWork Telework trendlines (2009),

http://www.workingfromanywhere.org/news/Trendlines 2009. pdf. 